\title{
Recherche des systèmes planétaires par accélérométrie absolue
}

\author{
P. Connes \\ Service d'Aéronomie du CNRS, B.P. 3, 91371 Verrières-le-Buisson cedex, France
}

\begin{abstract}
Résumé. Description d'une nouvelle méthode de mesure des petites variations de vitesses radiales stellaires. Le système associe un spectromètre à réseau, un Fabry-Perot d'épaisseur variable, un laser accordable et un laser stabilisé. Les résultats sont donnés par la variation d'une fréquence de battements.
\end{abstract}

\section{INTRODUCTION}

La seule méthode de recherche des systèmes planétaires extrasolaires ayant donné des résultats positifs est celle qui utilise le petit déplacement de l'étoile centrale autour du centre de gravité du système. Ce déplacement est détectable par la variation périodique du décalage Doppler dans les raies de l'étoile. Pour les quelques planètes détectées jusqu'ici, la vitesse induite de l'étoile était de l'ordre de $100 \mathrm{~m} / \mathrm{s}$, et la période de quelques semaines ou mois : elle était assez facilement mesurable par les moyens actuels. Mais on calcule que dans le cas (généralement pris comme typique d'un système plus ou moins semblable au nôtre) d'un Jupiter tournant autour d'un Soleil, la vitesse induite est de $13 \mathrm{~m} / \mathrm{s}$ et la période est de 12 ans. Pour la mesurer de façon satisfaisante, il faut donc que la précision des mesures, et en particulier leur stabilité à très long terme, soit de l'ordre de $1 \mathrm{~m} / \mathrm{s}$. C'est pour atteindre ce résultat que nous avons proposé la méthode baptisée Accélérométrie absolue, en cours de développement à Verrières et à l'Observatoire de Haute Provence.

Les difficultés sont de deux sortes. D'une part une erreur de $1 \mathrm{~m} / \mathrm{s}$ est très petite par rapport à la largeur des raies: celle-ci est de l'ordre de $6000 \mathrm{~m} / \mathrm{s}$ pour les raies solaires les plus fines. Le rapport signal/bruit est donc en général très faible. Le problème n'est soluble qu'aux conditions suivantes : 1) avoir un bon rendement dans la transmission des photons au détecteur 2) extraire toute l'information sur la vitesse radiale contenue dans le profil des raies 3) moyenner les résultats sur un grand nombre de raies. A partir de spectres stellaires typiques, et du nombre de photons reçus à partir d'une étoile de magnitude donnée, nous avons pu calculer l'erreur RMS minimum possible, due au seul bruit de photons. Le seul instrument qui permette d'approcher ce résultat est le spectrographe à réseau (à dispersion croisée par un prisme) muni d'un CCD.

La seconde difficulté vient de ce que l'erreur tolérée est également très petite par rapport aux déplacements systématiques de raies causés par les mouvements de la Terre: leurs valeurs maximum sont de l'ordre de $\pm 400 \mathrm{~m} / \mathrm{s}$ avec une période de 1 jour et $\pm 30 \mathrm{~km} / \mathrm{s}$ avec une période de 1 an. Ces déplacements sont calculables avec une précision suffisante, et peuvent en principe être soustraits des valeurs mesurées sur l'étoile : il n'en résulte donc aucune 
limitation fondamentale (contrairement au cas du bruit de photons), mais une difficulté pratique. Cependant, celle-ci est considérable: dans un cas typique, avec le spectrographe proposé plus haut, la largeur des raies correspondrait à 2 pixels, le déplacement maximum à 10 pixels et la précision cherchée à $3.10^{-4}$ pixel. Les méthodes de calibration usuelles sont incapables de donner cette précision en présence d'un déplacement aussi grand. Le procédé, habituellement employé, qui consiste à placer sur le faisceau issu du télescope une cuve à gaz absorbant, donc à imprimer sur le spectre stellaire quelques raies stables, permet d'éliminer le plus gros des dérives du spectromètre, donc de fixer le point zéro des mesures; il ne résoud pas le problème d'étendue dynamique qui se pose ici.

Le spectromètre à réseau résoud donc la première de nos deuxdifficultés, mais pas la seconde. On peut noter que la spectrométrie de Fourier fait exactement l'inverse: elle permet sans peine de mesurer des positions de raies quelconques, dans un spectre d'étendue arbitraire, avec la précision cherchée; l'emploi d'un laser stabilisé (par exemple sur l'iode) garantit la stabilité à très long terme. Mais, pour un spectre d'absorption dans le visible, le rapport signal/bruit ne peut approcher de celui obtenu à partir d'un spectre dispersé sur un CCD. Son emploi serait limité au cas de quelques étoiles très brillantes, observées avec un grand télescope.

L'accélérométrie absolue vise à résoudre les deux difficultés simultanément. Elle combine les points forts des deux méthodes: c'est un spectromètre à réseau (avec un CCD) qui traite le faisceau stellaire, mais c'est un système comprenant un interféromètre et deux lasers qui contrôle le spectromètre. Le terme d'accélérométrie est justifié parce que la méthode ne mesure que des variations de vitesses radiales (et non les vitesses elles-mêmes) ; elle est absolue parce que toute opération d'étalonnage est éliminée, et les résultats donnés directement à partir de mesures de fréquences.

\section{DESCRIPTION}

Le spectromètre peut recevoir soit le faisceau stellaire, soit celui d'une source de lumière blanche après traversée d' un étalon FP (de finesse très faible, de I'ordre de 2 à 3 ). La commutation est effectuée par un simple miroir pivotant, les durées de pose étant de l'ordre de quelques dizaines ou centaines de secondes. Au cours d'une première nuit, l'ordinateur enregistre une paire de spectres, dits de référence ; l'épaisseur du FP est quelconque, dans de larges limites. Ensuite les mesures de variations de vitesses radiales peuvent commencer. Pendant une séquence d'observation (de quelques minutes à quelques heures), les spectres stellaires et les spectres cannelés sont enregistrés alternativement. Après la prise de chaque paire, les deux spectres sont aussitôt comparés chacun à sa référence. Tous deux se sont décalés sur les pixels, en raison 1) de la dérive du spectromètre, 2) de la dérive du FP, 3) de la variation de vitesse radiale de l'étoile. L'ordinateur calcule aussitôt les variations d'épaisseur du FP et de réglage du réseau qui ramèneraient les spectres en coïncidence avec leurs références respectives. Deux boucles d'asservissement digitales appliquent aussitôt ces corrections ; après quoi un nouveau cycle est lancé. Le résultat est que les raies du spectre cannelé sont asservies à suivre en permanence les déplacements de celles du spectre stellaire. Le rôle du spectromètre est terminé.

Il reste à contrôler les variations d'épaisseur du FP, ce qui est fait par un procédé standard : le faisceau d'un laser accordable (de longueur d'onde quelconque, mais extérieure au domaine du spectre stellaire) traverse le FP et tombe sur un détecteur approprié. Une troisième boucle d'asservissement (purement analogique) asservit le laser à suivre une cannelure (quelconque) du FP. Lorsque ce résultat est atteint, notre problème de base (mesurer des déplacements de raies dans un spectre optique) a été transféré du domaine de l'optique incohérente à celui de l'optique cohérente : nous avons asservi une raie laser à suivre les déplacements de nos raies stellaires. Il ne reste plus qu'à mesurer les variations de fréquence de ce laser, lesquelles sont accessibles par celles des battements avec un laser stabilisé. 
Si $V_{R E F}$ et $V_{N}$ sont les vitesses radiales de l'étoile pendant la pose de référence et la pose $N$ respectivement, $F_{\text {REF }}$ et $F_{N}$ les fréquences de battements, $c$ la vitesse de la lumière et $F_{S T A B}$ la fréquence du laser stabilisé, on trouve que:

$$
\left(V_{N}-V_{R E F}\right) / c=\left(F_{R E F}-F_{N}\right) / F_{S T A B}
$$

Dans ce résultat, tous les intermédiaires ont disparu; ne figurent ni les longueurs d'onde, ni les profils des raies, ni l'épaisseur du FP, ni les paramètres du spectromètre ou du $\mathrm{CCD}$. On peut dire que l'ensemble spectromètre-CCD fonctionne en détecteur de zéro : grâce aux deux premières boucles d'asservissement, il n'a jamais à mesurer ce grand déplacement des raies (jusqu'à 10 pixels), mais seulement à annuler, à la fin de chaque cycle, les dérives résiduelles (une très petite fraction de pixel). La méthode présente bien toutes les caractéristiques d'unte méthode absolue, au sens habituel du terme en métrologie.

Un exposé détaillé du principe de la méthode est donné en [1], et de plus sommaires en [2] et [3]. L'état présent du développement est décrit en [4] et [5].

\section{Références}

[1] Connes P., Astrophys. Sp. Sc., 110, (1985), 211

[2] Connes P., IAU Coll., 88, (1984), p 131

[3] Connes P., IAU Symp., 112,(1985), p 91

[4] Connes P., Astrophys. Sp. Sc., 212, (1994), 357

[5] Connes P., Martic M., Schmitt J., Sp. Sc. Rev. (in print, 1997) 